\title{
Module character inner amenability of Banach algebras
}

\author{
H. Sadeghi ${ }^{1}$ M. Lashkarizadeh Bami ${ }^{2}$
}

Received: 19 March 2016/Accepted: 26 January 2017/Published online: 17 February 2017

(c) The Author(s) 2017. This article is published with open access at Springerlink.com

\begin{abstract}
In the present paper, we introduce the notion of module $(\phi, \varphi)$-inner amenability and module character inner amenability for a Banach algebra $A$ which is a Banach module over another Banach algebra $\mathfrak{A}$ with compatible actions. We characterize module $(\phi, \varphi)$-inner amenability and prove some hereditary properties.
\end{abstract}

Keywords Module $(\phi, \varphi)$-inner amenability $\cdot$ Module character inner amenability · Banach $\mathfrak{A}$-bimodule

\section{Mathematics Subject Classification $46 \mathrm{H} 25$}

\section{Introduction and preliminaries}

Lau [10] introduced a wide class of Banach algebras, called $F$-algebras, and studied the notion of left amenability for these algebras. In [12], Nasr-Isfahani introduced the concept of inner amenability for Lau algebras. A Lau algebra $A$ was said to be inner amenable if there exists a topological inner invariant mean on the $W^{*}$-algebra $A^{*}$, that is, a positive linear functional $m$ of norm 1 on $A^{*}$, such that $m(f . a)=m(a . f)$ for all $f \in A^{*}$ and all $a \in P_{1}(A)=\{a \in$

H. Sadeghi

h.sadeghi@iaufr.ac.ir

M. Lashkarizadeh Bami

lashkari@sci.ui.ac.ir

1 Department of Mathematics, Fereydan Branch, Islamic Azad University, Isfahan, Iran

2 Department of Mathematics, Faculty of Science, University of Isfahan, P.O. Box 81745-163, Isfahan, Iran
$A:\|a\|=1\}$ (or equivalently, for all $a \in A$ ). Commutative Lau algebras, such as the Fourier algebra $A(G)$ of a locally compact group $G$, are examples of inner amenable algebras. In addition, the group algebra $L^{1}(G)$ of any locally compact group $G$ is inner amenable.

Recently, Jabbari et al. [8] have introduced the notion of $\varphi$-inner amenability for a Banach algebra $A$, where $\varphi \in \Delta(A)$, the character space of A. A Banach algebra $A$ was said to be $\varphi$-inner amenable if there exists a $m \in A^{* *}$ satisfying $m(\varphi)=1$ and $m(f . a)=m(a . f)\left(a \in A, f \in A^{*}\right)$. $A$ is said to be character inner amenable if and only if $A$ is $\varphi$-inner amenable for every $\varphi \in \Delta(A)$.

In [6], Ebrahimi Vishki and Khoddami have investigated the character inner amenability for certain products of Banach algebras consist of projective tensor product $A \widehat{\otimes} B$, Lau product $A \times{ }_{\theta} B$, where $\theta \in \Delta(B)$ and the module extension $A \oplus X$. For instance, they showed that the projective tensor product $A \widehat{\otimes} B$ is character inner amenable if and only if both $A$ and $B$ are character inner amenable.

Let $\mathfrak{A}$ and $A$ be Banach algebras, such that $A$ be a Banach $\mathfrak{A}$-bimodule with compatible actions

$$
\begin{aligned}
& \alpha .(a b)=(\alpha . a) b,(a b) \cdot \alpha=a(b \cdot \alpha), \alpha \cdot(\beta . a)=(\alpha \beta) . a,(a . \beta) . \\
& \quad \alpha=a .(\beta \alpha)
\end{aligned}
$$

for all $a, b \in A$ and $\alpha \in \mathfrak{A}$.

Let $X$ be a Banach $A$-bimodule and a Banach $\mathfrak{A}$-bimodule with compatible left actions defined by

$$
\begin{aligned}
\alpha \cdot(a \cdot x)= & (\alpha \cdot a) \cdot x, a \cdot(\alpha \cdot x)=(a \cdot \alpha) \cdot x,(\alpha \cdot x) \cdot a=\alpha . \\
& (x \cdot a)(a \in A, \alpha \in \mathfrak{U}, \mathfrak{x} \in \mathfrak{X}),
\end{aligned}
$$

and similar for the right or two-sided actions. Then, we say that $X$ is a Banach $A$ - $\mathfrak{A}$-module. 
Let $A \widehat{\otimes} A$ be the projective tensor product of $A$ and $A$ which is a Banach $A$-bimodule and a Banach $\mathfrak{U}$-bimodule by the following actions:

$\alpha .(a \otimes b)=(\alpha . a) \otimes b, c .(a \otimes b)=(c a) \otimes b(\alpha \in \mathfrak{A}, \mathfrak{a}, \mathfrak{b}, \mathfrak{c} \in \mathfrak{U})$,

similarly for the right actions. Let $I_{A \widehat{\otimes A}}$ be the closed ideal of $A \widehat{\otimes} A$ generated by elements of the form:

$\{a . \alpha \otimes b-a \otimes \alpha . b \mid \alpha \in \mathfrak{U}, \mathfrak{a}, \mathfrak{b} \in \mathfrak{U}\}$.

Consider the map $\omega_{A} \in \mathcal{L}(A \widehat{\otimes} A, A)$ defined by $\omega_{A}(a \otimes$ $b)=a b$ and extended by linearity and continuity. Let $J_{A}$ be the closed ideal of $A$ generated by

$$
\omega\left(I_{A \widehat{\otimes A}}\right)=\{(a . \alpha) b-a(\alpha . b) \quad \mid a, b \in A, \alpha \in \mathfrak{Y}\} .
$$

Then, the module projective tensor product $A \widehat{\otimes}_{\mathfrak{A}} A$, which is $(A \widehat{\otimes} A) / I_{A \widehat{\otimes} A}$ by [16], and the quotient Banach algebra $A / J_{A}$ are both Banach $A$-bimodules and Banach $\mathfrak{U}$-bimodules. In addition, $A / J_{A}$ is $A$ - $\mathfrak{A}$-module with compatible actions when $A$ acts on $A / J_{A}$ canonically.

Let $\mathrm{A}$ and $\mathfrak{U}$ be Banach algebras, such that $\mathrm{A}$ is a Banach $\mathfrak{U}$-bimodule with compatible actions. Let $\varphi \in$ $\Delta(\mathfrak{U}) \cup\{\}$ and consider the set $\Omega_{A, \varphi}$ of linear continuous maps $\phi: A \rightarrow \mathfrak{U}$, such that

$$
\begin{aligned}
& \phi(a b)=\phi(a) \phi(b), \phi(\alpha . a)=\phi(a . \alpha)=\varphi(\alpha) \phi(a) \\
& (a, b \in A, \alpha \in \mathfrak{U}) .
\end{aligned}
$$

The concept of module $(\phi, \varphi)$-amenability and module character amenability for Banach algebra $A$, where $\varphi \in$ $\Delta(\mathfrak{U})$ and $\phi \in \Omega_{A}$ were introduced by Bodaghi and Amini in [4].

Our aim in this paper is to introduce and study module $(\phi, \varphi)$-inner amenability and module character inner amenability of Banach algebras. We characterize $(\phi, \varphi)$ inner amenability and prove some hereditary properties. Moreover, we investigate that module $(\phi, \varphi)$-inner amenability for certain class of Banach algebras consists of projective tensor product $A \widehat{\otimes} B, A \oplus_{\infty} B$, and $A \oplus_{p} B$, the $l^{p}$-direct sum of $A$ and $B$, where $1 \leq p<\infty$.

\section{Characterization and hereditary properties}

We commence this section with the following definition.

Definition 2.1 Let $A$ be a Banach $\mathfrak{U}$-bimodule and let $\varphi \in \Delta(\mathfrak{U})$ and $\phi \in \Omega_{A}$. Then, $A$ is called module $(\phi, \varphi)$ inner amenable if there exists $m \in A^{* *}$, such that $m(\varphi \circ \phi)=1, m(f . a)=m(a . f)$ and $m(\alpha . f)=m(f . \alpha)$ for all $a \in A, f \in A^{*}$ and $\alpha \in \mathfrak{U}$. A Banach $\mathfrak{U}$-bimodule $A$ is called module character inner amenable if it is module $(\phi, \varphi)$-inner amenable for each $\varphi \in \Delta(\mathfrak{U})$ and $\phi \in \Omega_{A}$.

We note that if $\mathfrak{U}=\mathbb{C}$ and $\varphi$ is the identity map, then the module $(\phi, \varphi)$-inner amenability and module character inner amenability coincide with $\phi$-inner amenability and character inner amenability (see [8] and [6]).

The next theorem characterizes module $(\phi, \varphi)$-inner amenability of Banach algebras that is analogue of Proposition 2.1 of [5] on module $(\phi, \varphi)$-amenable Banach algebras.

Theorem 2.2 Let $A$ be a Banach $\mathfrak{A}$-bimodule and let $\varphi \in \Delta(\mathfrak{U})$ and $\phi \in \Omega_{A}$. Then, the following statements are equivalent:

(i) A is module $(\phi, \varphi)$-inner amenable;

(ii) There exists a bounded net $\left(a_{i}\right)_{i}$ in $A$ such that $\left\|a a_{i}-a_{i} a\right\| \longrightarrow 0,\left\|\alpha . a_{i}-a_{i} . \alpha\right\| \longrightarrow 0(a \in A, \alpha \in$ $\mathfrak{U})$ and $\varphi \circ \phi\left(a_{i}\right)=1$ for all $i$;

(iii) There exists a bounded net $\left(a_{i}\right)_{i}$ in $A$ such that $\left\|a a_{i}-a_{i} a\right\| \longrightarrow 0,\left\|\quad \alpha . a_{i}-a_{i} \cdot \alpha\right\| \longrightarrow 0(a \in$ $A, \alpha \in \mathfrak{U})$ and $\varphi \circ \phi\left(a_{i}\right) \longrightarrow 1$.

Proof (iii) $\Rightarrow$ (i) Assume that a net $\left(a_{i}\right)_{i}$ exists. Let $m$ be a $w^{*}$-cluster point of the net $\left(a_{i}\right)_{i}$ in $A^{* *}$. Then, $\langle m, \varphi \circ \phi\rangle=$ $\lim _{i}\left\langle\varphi \circ \phi, a_{i}\right\rangle=1$. For every $a \in A$ and $f \in A^{*}$, we have

$$
\begin{aligned}
\langle m, f . a\rangle & =\lim _{i}\left\langle f . a, a_{i}\right\rangle=\lim _{i}\left\langle f, a a_{i}\right\rangle \\
& =\lim _{i}\left\langle f, a a_{i}-a_{i} a\right\rangle+\lim _{i}\left\langle f, a_{i} a\right\rangle \\
& =\lim _{i}\left\langle a . f, a_{i}\right\rangle=\langle m, a . f\rangle,
\end{aligned}
$$

and similarly, we have $\langle m, f . \alpha\rangle=\langle m, \alpha . f\rangle(\alpha \in \mathfrak{A})$. Therefore, $A$ is module $(\phi, \varphi)$-inner amenable.

(i) $\Rightarrow$ (ii) Suppose that $A$ is module $(\phi, \varphi)$-inner amenable. Then, there exists $m \in A^{* *}$ such that $m(\varphi \circ \phi)=1, m(f . a)=m(a . f)$, and $m(\alpha . f)=m(f . \alpha)$ for all $a \in A, f \in A^{*}$ and $\alpha \in \mathfrak{A}$. Choose a net $\left(u_{\beta}\right)_{\beta}$ in $A$ with $u_{\beta} \longrightarrow m$ in the $w^{*}$-topology of $A^{* *}$ and $\left\|u_{\beta}\right\| \leq\|m\|$ for all $\beta$. Since $\left\langle\varphi \circ \phi, u_{\beta}\right\rangle \longrightarrow\langle\varphi \circ \phi, m\rangle=1$, passing to a subnet and replacing $u_{\beta}$ by $\left(1 / \varphi \circ \phi\left(u_{\beta}\right)\right) u_{\beta}$, we may assume that $\varphi \circ \phi\left(u_{\beta}\right)=1$ and $\left\|u_{\beta}\right\| \leq\|m\|+1$ for all $\beta$. Consider the product space $A^{A}$ endowed with the product of norm topological. Define a linear map $T: A \rightarrow A^{A}$ by $T(b)=(a b-b a+\alpha . b-b . \alpha)_{a \in A}$, for all $b \in A$ and $\alpha \in \mathfrak{U}$. Let

$B=\{b \in A:\|b\| \leq\|m\|+1$ and $\varphi \circ \phi(\mathrm{b})=1\} \subseteq \mathrm{A}$.

Clearly, $B$ is convex and so $T(B)$ is a convex subset of $A^{A}$. For every $f \in A^{*}$, we have 
$\left\langle f, a u_{\beta}-u_{\beta} a+\alpha . u_{\beta}-u_{\beta} . \alpha\right\rangle$

$=\left\langle f, a u_{\beta}\right\rangle-\left\langle f, u_{\beta} a\right\rangle+\left\langle f, \alpha \cdot u_{\beta}\right\rangle+\left\langle f, u_{\beta} \cdot \alpha\right\rangle$

$=\left\langle f . a, u_{\beta}\right\rangle-\left\langle a . f, u_{\beta}\right\rangle+\left\langle f . \alpha, u_{\beta}\right\rangle+\left\langle\alpha . f, u_{\beta}\right\rangle$

$\rightarrow\langle m, f . a\rangle-\langle m, a . f\rangle+\langle m, f . \alpha\rangle-\langle m, \alpha . f\rangle$

$=0$.

This product of weak topologies coincide with topology on $A^{A}$ (see Theorem 4.3 of [17]). By Mazur's theorem, $0 \in$ $\overline{T(B)}^{w}=\overline{T(B)}^{\|\cdot\|}$. Therefore, there exists a bounded net $\left(a_{i}\right)_{i}$ in $A$, such that $\varphi \circ \phi\left(a_{i}\right)=1$ and

$\left\|a a_{i}-a_{i} a\right\| \longrightarrow 0,\left\|\alpha . a_{i}-a_{i} . \alpha\right\| \longrightarrow 0(a \in A, \alpha \in \mathfrak{U})$.

(ii) $\Rightarrow$ (iii) It is clear.

Definition 2.3 We say that the Banach algebra $\mathfrak{A}$ acts trivially on $A$ from the left (right) if there is a multiplicative linear functional $f$ on $\mathfrak{H}$, such that $\alpha . a=f(\alpha) a$ (resp. $a . \alpha=f(\alpha) a)$ for all $\alpha \in \mathfrak{U}$ and $a \in A$.

For the proof of the following result, we refer to Lemma 3.13 of [1].

Lemma 2.4 Let $\mathfrak{U}$ acts on A trivially from the left or right and $A / J_{A}$ has a right bounded approximate identity, then for each $\alpha \in \mathfrak{U}$ and $a \in A$ we have $f(\alpha) a-a . \alpha \in J_{A}$.

Let $\varphi \in \Delta(\mathfrak{H}) \quad$ and $\quad \phi \in \Omega_{A} . \quad$ Clearly, $\phi((a . \alpha) b-a(\alpha . b))=0(\alpha \in \mathfrak{U}, \mathfrak{a}, \mathfrak{b} \in \mathfrak{A})$, and hence, $\phi=$ 0 on $J_{A}$ and $\tilde{\phi}: A / J_{A} \longrightarrow \mathfrak{U}$ given by $\tilde{\phi}\left(a+J_{A}\right)=\phi(a)$ is well defined. Then, $\tilde{\phi} \in \Omega_{A / J_{A}}$.

Proposition 2.5 Let $A$ be a Banach $\mathfrak{X}$-bimodule, and let $\mathfrak{U}$ acts on A trivially from the left and $A / J_{A}$ has a bounded approximate identity. Then $A / J_{A}$ is $\varphi \circ \tilde{\phi}$-inner amenable for every $\varphi \in \Delta(\mathfrak{I})$ and $\phi \in \Omega_{A}$.

Proof Let $\left(e_{\alpha}+J_{A}\right)_{\alpha}$ be a bounded approximate identity of $A / J_{A}$ and let $\varphi \in \Delta(\mathfrak{U})$ and $\phi \in \Omega_{A}$. Then, $\varphi \circ \tilde{\phi}\left(e_{\alpha}+J_{A}\right) \longrightarrow 1$. Clearly

$\left\|\left(a+J_{A}\right)\left(e_{\alpha}+J_{A}\right)-\left(e_{\alpha}+J_{A}\right)\left(a+J_{A}\right)\right\| \longrightarrow 0$

for all $a+J_{A} \in A / J_{A}$. By Proposition 2.2 of [6], $A / J_{A}$ is $\varphi \circ \tilde{\phi}$-inner amenable.

Note that in the above proposition, both left and right actions of $\mathfrak{U}$ on $A / J_{A}$ are trivial, by Lemma 2.4. Therefore, for every $\alpha \in \mathfrak{U}$, we have

$$
\begin{aligned}
& \left\|\alpha .\left(e_{\alpha}+J_{A}\right)-\left(e_{\alpha}+J_{A}\right) \cdot \alpha\right\| \\
& =\left\|\alpha . e_{\alpha}+J_{A}-e_{\alpha} \cdot \alpha+J_{A}\right\| \\
& =\left\|f(\alpha)\left(e_{\alpha}+J_{A}\right)-f(\alpha)\left(e_{\alpha}+J_{A}\right)\right\|=0 .
\end{aligned}
$$

Then, the net $\left(e_{\alpha}+J_{A}\right)_{\alpha}$ satisfies condition (iii) of Theorem 2.2 , and hence, $A / J_{A}$ is module $(\tilde{\phi}, \varphi)$-inner amenable.
Remark 2.6 A inverse semigroup is a discrete semigroup $S$, such that for each $s \in S$, there is a unique element $s^{*} \in S$ with $s s^{*} s=s$ and $s^{*} s s^{*}=s^{*}$. An element $e \in S$ is called an idempotent if $e^{2}=e^{*}=e$. The set of idempotent elements of $S$ is denoted by $E_{S}$. Define the relation $\leq$ on $E_{S}$ by $e \leq d \Leftrightarrow e d=e\left(e, d \in E_{S}\right)$. Then, $E_{S}$ is a commutative subsemigroup of $S$, and $l^{1}\left(E_{S}\right)$ may be regarded as a subalgebra of $l^{1}(S)$.

Let $s$ be an inverse semigroup with the set of idempotents $E_{S}$. We let $l^{1}\left(E_{S}\right)$ acts on $l^{1}(S)$ by multiplication from the right and trivially from the left, that is

$\delta_{e} . \delta_{s}=\delta_{s} \delta_{s} . \delta_{e}=\delta_{s e}=\delta_{s} * \delta_{e}\left(e \in E_{S}, s \in S\right)$.

By these actions, $l_{1}(S)$ becomes a Banach $l_{1}\left(E_{S}\right)$-module. In this case

$J_{l^{1}(S)}=\left\{\delta_{s e t}-\delta_{s t} \mid e \in E_{S}, s, t \in S\right\}$.

We consider an equivalence relation on $S$ as follows $s \approx$ $t \Leftrightarrow \delta_{s}-\delta_{t} \in J_{l^{1}(S)}(s, t \in S)$. For inverse semigroup $S$, the quotient semigroup $S / \approx$ is discrete group and so $l^{1}(S / \approx)$ has an identity (see $[2,13]$ ). Indeed, $S / \approx$ is homomorphic to the maximal group homomorphic image $G_{S}$ of $S$ (see $[11,14])$. It is also shown in Theorem 3.3 of [15] that $l^{1}(S) / J_{l^{1}(S)} \cong l^{1}(S / \approx)=l^{1}\left(G_{S}\right)$ is a commutative $l^{1}\left(E_{S}\right)$ bimodule with the following actions:

$\delta_{e} . \delta_{[s]}=\delta_{[s]}, \delta_{[s]} . \delta_{e}=\delta_{[s e]}\left(s \in S, e \in E_{S}\right)$,

where $[s]$ denotes the equivalence class of $s$ in $G_{S}$.

It is shown in [4] that the maps $\varphi$ and $\phi$ satisfying (3) exist for $l^{1}(S)$.

Example 2.7 Let $S$ be an inverse semigroup with the set of idempotents $E_{S}$. Consider $l^{1}(S)$ as a Banach module over $l^{1}\left(E_{S}\right)$ with the trivial left action and natural right action. Then, by Proposition $2.5, l^{1}\left(G_{S}\right)$ is $\varphi \circ \tilde{\phi}$-inner amenable (module $(\tilde{\phi}, \varphi)$-inner amenable) for all $\varphi \in \Delta\left(l^{1}\left(E_{S}\right)\right)$ and $\phi \in \Omega_{l^{1}(S)}$.

Example 2.8 Let $A$ be a commutative Banach algebra and commutative $\mathfrak{U}$-bimodule (i.e., $\alpha . a=a . \alpha(a \in A, \alpha \in \mathfrak{U}))$. Let $\varphi \in \Delta(\mathfrak{U}), \phi \in \Omega_{\mathfrak{A}}$ and let $a \in A$ be such that $\varphi \circ \phi(a)=1$. put $m=\widehat{a}$. Then, $m(\varphi \circ \phi)=\widehat{a}(\varphi \circ \phi)=$ $\varphi \circ \phi(a)=1$ and clearly, $m(f . a)=m(a . f)$ and $m(\alpha . f)=$ $m(f . \alpha)$ for all $a \in A, \alpha \in \mathfrak{U}$. Therefore, $A$ is module $(\phi, \varphi)$ inner amenable. In particular, if $S$ is a commutative inverse semigroup, then $l^{1}(S)$ is commutative and commutative $l^{1}\left(E_{S}\right)$-bimodule. Therefore, $l^{1}(S)$ is module $(\phi, \varphi)$-inner amenable for all $\varphi \in \Delta\left(l^{1}\left(E_{S}\right)\right)$ and $\phi \in \Omega_{l^{1}(S)}$.

Example 2.9 Let $S=(\mathbb{N}, \wedge)$ be the inverse semigroup of positive integers with the minimum operation. Let $A=$ $l^{1}(S), \mathfrak{U}=\mathfrak{I}\left(\mathfrak{E}_{\mathfrak{S}}\right)$ and $\mathfrak{U}$ acts on $A$ by the following actions: 


$$
\delta_{e} . \delta_{s}=\delta_{s} . \delta_{e}=\delta_{s e} \quad\left(e \in E_{S}, s \in S\right) .
$$

$A$ is module amenable (see page 42 of [3]). By Theorem 2.1 of [4], $A$ is module character amenable. Let $\varphi \in \Delta(\mathfrak{U})$ and $\phi \in \Omega_{A}$. Then, there exist $m \in A^{* *}$, such that $m(f . a)=$ $\varphi \circ \phi(a) m(f), m(f . \alpha)=\varphi(\alpha) m(f)$ and $m(\varphi \circ \phi)=1$ for every $f \in A^{*}, a \in A$ and $\alpha \in \mathfrak{U}$. Let $\left(a_{\alpha}\right)_{\alpha}$ be a net in $A$ converging to $m$ in the $w^{*}$-topology of $A^{* *}$. Since $A$ is commutative and commutative $\mathfrak{U}$-bimodule, for every $a \in A, f \in A^{*}$

$$
\begin{aligned}
m(f . a) & =\lim _{\alpha}\left\langle f . a, a_{\alpha}\right\rangle=\lim _{\alpha}\left\langle f, a a_{\alpha}\right\rangle \\
& =\lim _{\alpha}\left\langle f, a_{\alpha} a\right\rangle=\lim _{\alpha}\left\langle a . f, a_{\alpha}\right\rangle \\
& =m(a . f) .
\end{aligned}
$$

Similarly, for every $\alpha \in \mathfrak{A}$ and $f \in A^{*}$, we have

$m(f . \alpha)=m(\alpha . f)$.

Thus, $A$ is module $(\phi, \varphi)$-inner amenable. Therefore, $A$ is module character inner amenable.

The proof of the following proposition is adapted from that of Proposition 2.3 of [4].

Proposition 2.10 Let $A$ and $B$ be Banach $\mathfrak{A}$-bimodules and let $h$ be an $\mathfrak{U}$-module homomorphism with dense range. If $\phi \in \Omega_{B}, \varphi \in \Delta(\mathfrak{U})$ and $A$ is module $(\phi \circ h, \varphi)$ inner amenable, then $B$ is module $(\phi, \varphi)$-inner amenable.

Proof Let $m \in A^{* *}$ be such that $m(\varphi \circ(\phi \circ h))=$ $1, m(f . a)=m(a . f)$ and $m(f . \alpha)=m(\alpha . f)$ for all $a \in A, f \in$ $A^{*} \quad$ and $\quad \alpha \in \mathfrak{A}$. Define $m_{B} \in B^{* *}$ by $m_{B}(g)=m(g \circ h)\left(g \in B^{*}\right)$. We show that $m_{B}(g . b)=$ $m_{B}(b . g)(b \in B)$. For see this let $b \in B$ be such that $h(a)=b$. One can easily check that $(g . h(a)) \circ h=(g \circ$ $h) . a$ and $(h(a) . g) \circ h=a .(g \circ h)$. Hence for every $g \in B^{*}$

$$
\begin{aligned}
m_{B}(g . b) & =m_{B}(g \cdot h(a))=m((g \cdot h(a)) \circ h) \\
& =m((g \circ h) \cdot a)=m(a \cdot(g \circ h)) \\
& =m((h(a) \cdot g) \circ h)=m_{B}(h(a) \cdot g) \\
& =m_{B}(b \cdot g) .
\end{aligned}
$$

By density of the range of $h$ and the continuity of $h$, we conclude that $m_{B}(g . b)=m_{B}(b . g)(b \in B)$. In addition, for every $\alpha \in \mathfrak{U}$, we have

$$
\begin{aligned}
m_{B}(g . \alpha) & =m((g . \alpha) \circ h)=m((g \circ h) . \alpha) \\
& =m(\alpha .(g \circ h))=m((\alpha . g) \circ h)) \\
& =m_{B}(\alpha . g) .
\end{aligned}
$$

Furthermore, $\quad m_{B}(\varphi \circ \phi)=m((\varphi \circ \phi) \circ h)=m(\varphi \circ(\phi \circ$ $h)=1$. Therefore, $B$ is module $(\phi, \varphi)$-inner amenable.
Corollary 2.11 Let $A$ and $B$ be Banach $\mathfrak{A}$-bimodules and let $h$ be an $\mathfrak{U}$-module homomorphism with dense range. Then the module character inner amenability of A implies the module character inner amenability of $B$. In particular, if $A$ is module character inner amenable, then so is $A / J_{A}$.

The proof idea of the following result is taken from the proof of Lemma 2.6 of [4].

Proposition 2.12 Let $A$ be a Banach $\mathfrak{U}$-bimodule and $I$ be a closed ideal and $\mathfrak{U}$-submodule of $A$, and let $\varphi \in \Delta(\mathfrak{U})$ and $\phi \in \Omega_{A}$ be such that $\left.\phi\right|_{I} \neq 0$. If $A$ is module $(\phi, \varphi)$ inner amenable, then $I$ is module $\left(\left.\phi\right|_{I}, \varphi\right)$-inner amenable.

Proof Let $m \in A^{* *} \quad$ satisfy $m(\varphi \circ \phi)=1, \quad m(f . a)=$ $m(a . f)$ and $m(\alpha . f)=m(f . \alpha)$ for all $a \in A, f \in A^{*}$ and $\alpha \in \mathfrak{U}$. By a similar argument as in the proof of Lemma 3.1 of [9], one can define a bounded linear functional $n$ on $I^{*}$ by, $n(g)=m(f)$ for all $g \in I^{*}$, where $f$ is an arbitrary element of $A^{*}$ extending $g$. Now, for every $g \in I^{*}, a \in I$ and $\alpha \in \mathfrak{U}$, we have

$n(g . a)=m(f . a)=m(a . f)=n(a . g)$,

and

$n(g . \alpha)=m(f . \alpha)=m(\alpha . f)=n(\alpha . g)$.

In addition, $n\left(\left.\varphi \circ \phi\right|_{I}\right)=m(\varphi \circ \phi)=1$. Therefore, $I$ is module $\left(\left.\phi\right|_{I}, \varphi\right)$-inner amenable.

We need to recall the following remark from [4] to give the next result:

Remark 2.13 Let $\mathfrak{U}$ be a Banach algebra and $\mathfrak{U}$ be the unitization of $\mathfrak{U}$ which is $\mathfrak{U}=\mathfrak{U} \oplus \mathbb{C}$ is a unital Banach algebra which contains $\mathfrak{U}$ as a closed ideal. Let $A$ be a Banach $\mathfrak{U}$-module. Then, $A$ is a Banach $\mathfrak{U}$-bimodule with the following module actions:

$(\alpha, \lambda) \cdot a=\alpha \cdot a+\lambda a, a \cdot(\alpha, \lambda)=a \cdot \alpha+\lambda a(\lambda \in \mathbb{C}, \alpha \in \mathfrak{U}, \mathfrak{a} \in \mathfrak{U})$.

Let $A^{\sharp}=(A \oplus \mathfrak{U}, \cdot)$, where the multiplication · is defined through

$(a, u) \cdot(b, v)=(a b+a . v+u . b, u v)(a, b \in A, u, v \in \mathfrak{U})$.

Then, with the actions defined by

$u .(a, v)=(u \cdot a, u v),(a, v) \cdot u=(a . u, v u)(a \in A, u, v \in \mathfrak{U})$,

$A^{\sharp}$ is a unital $\mathfrak{U}$-module Banach algebra with the identity $e_{A^{\sharp}}=\left(0, e_{\mathfrak{I}}\right)$, where $e_{\mathfrak{A}}=(0,1)$. Now, suppose that $\phi \in$ $\Omega_{A}$ and $\varphi^{\#}$ is the extension of $\varphi$ on $\mathfrak{U}$ defined by $\varphi^{\#}(\alpha, \lambda)=\varphi(\alpha)+\lambda(a \in A, \alpha \in \mathfrak{U}, \lambda \in \mathbb{C}$. If $u=(\alpha, \lambda)$ $\in \mathfrak{U}$, it is easy to see that 
$\phi(a . u)=\phi(u . a)=\varphi^{\#}(u) \phi(a)(a \in A)$.

Define $\phi^{\sharp}: A^{\sharp} \longrightarrow \mathfrak{U}$ by

$\phi^{\sharp}(a, u)=\left(\phi(a), \varphi^{\#}(u)\right)(a \in A, u \in \mathfrak{U})$.

Using (4), one can show that $\phi^{\sharp}$ is multiplicative and

$\phi^{\sharp}(u \cdot(a, v))=\phi^{\sharp}((a, v) \cdot u)=\varphi^{\#}(u) \phi^{\sharp}(a, v)(a \in A, u, v \in \mathfrak{U})$.

Therefore, $\phi^{\sharp}$ is an extension of $\phi$, such that $\phi^{\sharp}(0, u)=$ $\varphi^{\#}(u)$ is the extension $h_{0}=\tilde{0}$ of the zero function given by (5).

The proof of the following theorem is inspired by the proof of Proposition 2.7 of [4].

Proposition 2.14 Let $A$ be a Banach $\mathfrak{A}$-bimodule, and let $\varphi \in \Delta(\mathfrak{A})$ and $\phi \in \Omega_{A}$. Then, $A$ is module $(\phi, \varphi)$-inner amenable if and only if $A^{\sharp}$ is module $\left(\phi^{\sharp}, \varphi^{\#}\right)$-inner amenable.

Proof Let $A^{\sharp}$ be module $\left(\phi^{\sharp}, \varphi^{\#}\right)$-inner amenable. Since the image of $\left.\phi^{\sharp}\right|_{A}$ is included in $\mathfrak{U}$, by 2.12 , we conclude that $A$ is module $(\phi, \varphi)$-inner amenable.

Conversely, suppose that $A$ is module $(\phi, \varphi)$-inner amenable. Then, there exists a $m \in A^{* *}$, such that $m(\varphi \circ \phi)=1, m(f . a)=m(a . f)$, and $m(\alpha . f)=m(f . \alpha)$ for all $a \in A, f \in A^{*}$ and $\alpha \in \mathfrak{U}$. By Remark 2.13, we may identify the dual space $\left(A^{\sharp}\right)^{*}$ with $A^{*} \oplus \mathbb{C} h_{0}$, where $\left.h_{0}\right|_{A}=$ 0 and $h_{0}\left(e_{A^{\sharp}}\right)=1$. Define $n \in\left(A^{\sharp}\right)^{* *}$ by $n(f)=m(f)(f \in$ $\left.A^{*}\right)$ and $n\left(h_{0}\right)=0$. Since $A$ is an ideal and $\mathfrak{X}$-submodule of $A^{\sharp}$, it follows that $h_{0} \cdot a=0$ and $h_{0} \cdot \alpha=0$ for all $a \in A$ and $\alpha \in \mathfrak{U}$. A simple computation shows that

$n\left(\left(f+\lambda h_{0}\right) \cdot\left(a+\lambda^{\prime} e_{A^{\sharp}}\right)\right)=n\left(\left(a+\lambda^{\prime} e_{A^{\sharp}}\right) \cdot\left(f+\lambda h_{0}\right)\right)$,

and

$n\left(\left(f+\lambda h_{0}\right) \cdot u\right)=n\left(u \cdot\left(f+\lambda h_{0}\right)\right)$

for all $f \in A^{*}, a \in A, u \in \mathfrak{U}$ and $\lambda, \lambda^{\prime} \in \mathbb{C}$. For $f \in A^{*}$, consider the map $\bar{f}: A^{\sharp} \longrightarrow \mathbb{C}$ defined by $\bar{f}(a, u)=f(a)+$ $\tilde{\varphi}(u)(a \in A, u \in \mathfrak{U})$. Thus, $\varphi^{\#} \circ \phi^{\sharp}=\overline{\varphi \circ \phi}$, and hence

$n\left(\varphi^{\#} \circ \phi^{\sharp}\right)=n(\overline{\varphi \circ \phi})=n\left(\varphi \circ \phi+\lambda h_{0}\right)=m(\varphi \circ \phi)=1$.

Therefore, $A^{\sharp}$ is module $\left(\phi^{\sharp}, \varphi^{\#}\right)$-inner amenable.

\section{Module inner amenability of certain Banach algebras}

Let $A \widehat{\otimes} B$ be the projective tensor product of two Banach algebras $A$ and $B$. For every $f \in A^{*}$ and $g \in B^{*}$, let $f \otimes g$ denote the element of $(A \widehat{\otimes} B)^{*}$ satisfying, $f \otimes g(a \otimes b)=f(a) g(b)(a \in A, b \in B)$. In addition, note that $A \widehat{\otimes} B$ is a Banach $\mathfrak{U} \widehat{\otimes} \mathfrak{U}$-bimodule with the following actions:

$(\alpha \otimes \beta) .(a \otimes b)=(\alpha . a) \otimes(\beta . b)(a \in A, b \in B, \alpha, \beta \in \mathfrak{U})$,

and similarly for right action. For $\varphi_{1}, \varphi_{2} \in \Delta(\mathfrak{U}), \psi \in$ $\Omega_{\mathfrak{A}}\left(=\Omega_{\mathfrak{A}, \varphi}\right) \quad$ and $\quad \phi \in \Omega_{A}\left(=\Omega_{A, \varphi_{2}}\right)$, define $(\phi \otimes \psi)$ : $A \widehat{\otimes} \boldsymbol{B} \rightarrow \mathfrak{U} \widehat{\otimes} \mathfrak{U}$

$(\phi \otimes \psi)(a \otimes b)=\phi(a) \otimes \psi(b)(a \in A, b \in B) . \quad$ Clearly, $\phi \otimes \psi \in \Omega_{A \widehat{\otimes B}}\left(=\Omega_{A \widehat{\otimes B}, \varphi_{1} \otimes \varphi_{2}}\right)$ and $\varphi_{1} \otimes \varphi_{2} \in \Delta(\mathfrak{\mathcal { U }} \widehat{\otimes} \mathfrak{U})$. In addition, if $\bar{\varphi} \in \Delta(\mathfrak{U} \widehat{\otimes} \mathfrak{U})$, then $\bar{\varphi}=\varphi_{1} \otimes \varphi_{2}$, where $\varphi_{1}, \varphi_{2} \in \Delta(\mathfrak{U})$ (see [4]).

The technique of proof of the following theorem (one side) is similar to that of Theorem 2.8 of [4].

Theorem 3.1 Let $A$ and $B$ be Banach $\mathfrak{U}$-bimodules, and let $\varphi_{1}, \varphi_{2} \in \Delta(\mathfrak{U})$ and $\phi \in \Omega_{A}, \psi \in \Omega_{B}$. Then $A \widehat{\otimes} B$ is module $\left(\phi \otimes \psi, \varphi_{1} \otimes \varphi_{2}\right)$-inner amenable (as $\mathfrak{U} \widehat{\otimes} \mathfrak{U}$ module) if and only if $A$ is module $\left(\phi, \varphi_{1}\right)$-inner amenable and $B$ is module $\left(\psi, \varphi_{2}\right)$-inner amenable.

Proof Suppose that $A \widehat{\otimes} B$ is module $\left(\phi \otimes \psi, \varphi_{1} \otimes \varphi_{2}\right)$ inner amenable. Then, there exists $m \in(A \widehat{\otimes} B)^{* *}$, such that $m\left(\left(\varphi_{1} \otimes \varphi_{2}\right) \circ(\phi \otimes \psi)\right)=1$ and

$$
\begin{aligned}
m(f \otimes g .(a \otimes b)) & =m((a \otimes b) . f \otimes g), m(f \otimes g .(\alpha \otimes \beta)) \\
& =m((\alpha \otimes \beta) . f \otimes g),
\end{aligned}
$$

for all $a \otimes b \in A \widehat{\otimes} B, f \in A^{*}, g \in B^{*}$ and $\alpha \otimes \beta \in \mathfrak{U} \widehat{\otimes} \mathfrak{U}$. Define $m_{A}: A^{*} \rightarrow \mathbb{C}$ by $m_{A}(f)=m\left(f \otimes\left(\varphi_{2} \circ \psi\right)\right)\left(f \in A^{*}\right)$. Therefore, $m_{A}\left(\varphi_{1} \circ \phi\right)=m\left(\left(\varphi_{1} \circ \phi\right) \otimes\left(\varphi_{2} \circ \psi\right)\right)=m\left(\left(\varphi_{1}\right.\right.$ $\left.\left.\otimes \varphi_{2}\right) \circ(\phi \otimes \psi)\right)=1$. Choose $b_{0} \in A$, such that $\varphi_{2}$ $\circ \psi\left(b_{0}\right)=1$. Therefore, for every $a \in A$ and $f \in A^{*}$, we have

$$
\begin{aligned}
m_{A}(f . a) & =m\left((f . a) \otimes\left(\varphi_{2} \circ \psi\right)\right) \\
& =m\left((f . a) \otimes\left(\varphi_{2} \circ \psi\right) \cdot b_{0}\right) \\
& =m\left(f \otimes\left(\varphi_{2} \circ \psi\right) .\left(a \otimes b_{0}\right)\right) \\
& =m\left(\left(a \otimes b_{0}\right) . f \otimes\left(\varphi_{2} \circ \psi\right)\right) \\
& =m\left((a . f) \otimes b_{0} \cdot\left(\varphi_{2} \circ \psi\right)\right) \\
& =m_{A}(a . f) .
\end{aligned}
$$

Similarly, for every $\alpha \in \mathfrak{U}$, if we take $\beta \in \mathfrak{U}$, such that $\varphi_{2}(\beta)=1$, then

$$
\begin{aligned}
m_{A}(f . \alpha) & =m\left((f . \alpha) \otimes\left(\varphi_{2} \circ \psi\right)\right) \\
& =m\left((f . \alpha) \otimes\left(\varphi_{2} \circ \psi\right) . \beta\right) \\
& =m\left(f \otimes\left(\varphi_{2} \circ \psi\right) \cdot(\alpha \otimes \beta)\right) \\
& =m\left((\alpha \otimes \beta) . f \otimes\left(\varphi_{2} \circ \psi\right)\right) \\
& =m\left((\alpha . f) \otimes \beta .\left(\varphi_{2} \circ \psi\right)\right) \\
& =m\left((\alpha . f) \otimes\left(\varphi_{2} \circ \psi\right)\right) \\
& =m_{A}(\alpha . f),
\end{aligned}
$$


for all $f \in A^{*}$. Therefore, $A$ is module $\left(\varphi, \phi_{1}\right)$-inner amenable. Similarly, one can prove that $B$ is module $\left(\psi, \phi_{2}\right)$-inner amenable.

For the converse, let $A$ is module $\left(\phi, \varphi_{1}\right)$-inner amenable and $B$ is module $\left(\psi, \varphi_{2}\right)$-inner amenable. Then, by Theorem 2.2 , there exist bounded nets $\left(a_{i}\right)_{i}$ in $A$ and $\left(b_{j}\right)_{j}$ in $B$ with bounds $M_{1}$ and $M_{2}$, respectively, such that

$$
\begin{gathered}
\varphi_{1} \circ \phi\left(a_{i}\right)=1,\left\|a a_{i}-a_{i} a\right\| \longrightarrow 0,\left\|\alpha \cdot a_{i}-a_{i} \cdot \alpha\right\| \longrightarrow \\
0(a \in A, \alpha \in \mathfrak{U}),
\end{gathered}
$$

and

$$
\begin{aligned}
\varphi_{2} \circ \psi\left(b_{j}\right)= & 1,\left\|b b_{j}-b_{j} b\right\| \longrightarrow 0,\left\|\alpha \cdot b_{j}-b_{j} \cdot \alpha\right\| \\
& \longrightarrow 0(b \in B, \alpha \in \mathfrak{U}) .
\end{aligned}
$$

Consider the bounded net $\left(a_{i} \otimes b_{j}\right)_{(i, j)}$ in $A \widehat{\otimes} B$. Therefore, $\left(\left(\varphi_{1} \otimes \varphi_{2}\right) \circ(\phi \otimes \psi)\right)\left(a_{i} \otimes b_{j}\right)=\varphi_{1} \circ \phi\left(a_{i}\right) \varphi_{2} \circ \quad \psi\left(b_{j}\right)=$ 1. Let $\mathfrak{F}=\sum_{\mathrm{I}=}^{\mathfrak{N}} \alpha_{\mathrm{I}} \otimes \beta_{\mathrm{I}} \in \mathfrak{U} \widehat{\otimes} \mathfrak{U}$, then

$$
\begin{aligned}
& \left\|\mathfrak{F} \cdot\left(a_{i} \otimes b_{j}\right)-\left(a_{i} \otimes b_{j}\right) \cdot \mathfrak{F}\right\| \\
& \quad=\left\|\sum_{l=1}^{N}\left[\left(\alpha_{l} \cdot a_{i}-a_{i} \cdot \alpha_{l}\right) \otimes \beta_{l} \cdot b_{j}+a_{i} \cdot \alpha_{l} \otimes\left(\beta_{l} \cdot b_{j}-b_{j} \cdot \beta_{l}\right)\right]\right\| \\
& \quad \leq \sum_{l=1}^{N} M_{2}\left\|\beta_{l}\right\|\left\|\alpha_{l} \cdot a_{i}-a_{i} \cdot \alpha_{l}\right\|+\sum_{l=1}^{N} M_{1}\left\|\alpha_{l}\right\|\left\|\beta_{l} \cdot b_{j}-b_{j} \cdot \beta_{l}\right\| \longrightarrow 0 .
\end{aligned}
$$

Now, let $\mathfrak{G} \in \mathfrak{U} \widehat{\otimes} \mathfrak{A}$, so there exist sequences $\left(\alpha_{l}\right)_{l} \subseteq \mathfrak{U}$ and $\left(\beta_{l}\right)_{l} \subseteq \mathfrak{H}, \quad$ such that $\quad \mathfrak{F}=\sum_{\mathrm{I}=}^{\infty} \alpha_{\mathrm{I}} \otimes \beta_{\mathrm{I}} \quad$ with $\sum_{l=1}^{\infty}\left\|\alpha_{l}\right\|\left\|\beta_{l}\right\|<\infty$. By the same argument as in the proof of the Theorem 3.1 of [6], for every $\mathfrak{G} \in \mathfrak{U} \widehat{\otimes} \mathfrak{A}$, one can show that $\| \mathfrak{6} .\left(a_{i} \otimes b_{j}\right)-\left(a_{i} \otimes b_{j}\right) \cdot(\mathfrak{5} \| \longrightarrow 0$. Similarly, we may show that $\left\|G\left(a_{i} \otimes b_{j}\right)-\left(a_{i} \otimes b_{j}\right) G\right\| \longrightarrow 0$ for all $G \in A \widehat{\otimes} B$. Therefore, Proposition 2.2 implies that $A \widehat{\otimes} B$ is module $\left(\phi \otimes \psi, \varphi_{1} \otimes \varphi_{2}\right)$-inner amenable.

Let $A$ and $B$ be Banach algebras, it is well known that $A \oplus_{\infty} B$ and $A \oplus_{p} B$, the $l^{p}$-direct sum of $A$ and $B$, are Banach algebras with respect to the canonical multiplication defined by

$$
(a, b)(c, d):=(a c, b d) \quad(a, c \in A, b, d \in B),
$$

and norms $\|(a, b)\|=\max \{\|a\|,\|b\|\}$ and $\|(a, b)\|=$ $\left(\|a\|^{p}+\|b\|^{p}\right)^{\frac{1}{p}}(a \in A, b \in B)$. Furthermore, if $A$ and $B$ are two Banach $\mathfrak{X}$-bimodules, then $A \oplus_{\infty} B$ and $A \oplus_{p} B$ are Banach $\mathfrak{U}$-bimodules under the module actions

$$
\begin{aligned}
\alpha .(a, b) & =(\alpha . a, \alpha . b), \quad(a, b) \cdot \alpha \\
& =(a . \alpha, b \cdot \alpha) \quad(a \in A, b \in B, \alpha \in \mathfrak{U}) .
\end{aligned}
$$

Before stating the next theorem, we note that if for every $\varphi \in \Delta(\mathfrak{U}), \phi \in \Omega_{\mathfrak{Q}}$ and $\psi \in \Omega_{B}$, we define $(0, \psi): A \oplus_{p}$ $B \rightarrow \mathfrak{U}$ and $(\phi, 0): A \oplus_{p} B \rightarrow \mathfrak{U}$ by
$(0, \psi)(a, b)=\psi(b),(\phi, 0)(a, b)=\phi(a)(a \in A, b \in B)$,

where $\quad 1 \leq p \leq \infty$, then $(0, \psi) \quad$ and $\quad(\phi, 0) \in$ $\Omega_{A \oplus_{p} B}\left(=\Omega_{A \oplus_{p} B, \varphi}\right)$.

Theorem 3.2 Let $A$ and $B$ be two $\mathfrak{A}$-bimodule Banach algebras, $\varphi \in \Delta(\mathfrak{U}), \phi \in \Omega_{A}, \psi \in \Omega_{B} \quad$ and $\quad 1 \leq p \leq \infty$. Then the following statements are valid:

(i) $\quad A \oplus_{p} B$ is module $((\phi, 0), \varphi)$-inner amenable if and only if $A$ is module $(\phi, \varphi)$-inner amenable.

(ii) $\quad A \oplus_{p} B$ is module $((0, \psi), \varphi)$-inner amenable if and only if $B$ is module $(\psi, \varphi)$-inner amenable.

Proof (i) Assume that $A \oplus_{p} B$ is module $((\phi, 0), \varphi)$-inner amenable. By Theorem 2.2, there exists a net $\left(a_{i}, b_{i}\right)_{i}$ in $A \oplus_{p} B$, such that $\varphi \circ(\phi, 0)\left(a_{i}, b_{i}\right) \longrightarrow 1$ and

$$
\begin{aligned}
& \left\|(a, b) .\left(a_{i}, b_{i}\right)-\left(a_{i}, b_{i}\right) \cdot(a, b)\right\| \longrightarrow 0, \| \alpha \cdot\left(a_{i}, b_{i}\right) \\
& \quad-\left(a_{i}, b_{i}\right) \cdot \alpha \| \longrightarrow 0
\end{aligned}
$$

for all $(a, b) \in A \oplus_{p} B$ and $\alpha \in \mathfrak{A}$. Consider the bounded net $\left(a_{i}\right)_{i}$ in $A$. One can easily show that $\left\|a a_{i}-a_{i} a\right\| \longrightarrow 0$ and $\left\|\alpha . a_{i}-a_{i} . \alpha\right\| \longrightarrow 0$ for all $a \in A, \alpha \in \mathfrak{U}$. In addition, it is clear that $\varphi \circ \phi\left(a_{i}\right) \longrightarrow 1$. Therefore, Theorem 2.2 implies that $A$ is module $(\phi, \varphi)$-inner amenable.

Conversely, suppose that $A$ is module $(\phi, \varphi)$-inner amenable. Then, there exists a bounded net $\left(a_{i}\right)_{i}$ in $A$, such that $\varphi \circ \phi\left(a_{i}\right) \longrightarrow 1,\left\|a a_{i}-a_{i} a\right\| \longrightarrow 0$ and $\left\|\alpha . a_{i}-a_{i} . \alpha\right\|-$ $\rightarrow 0$ for all $a \in A, \alpha \in \mathfrak{U}$. Clearly, the bounded net $\left(a_{i}, 0\right)_{i} \subset A \oplus_{p} B$ satisfies in the condition (iii) of Theorem 2.2. Therefore, $A \oplus_{p} B$ is module $((\phi, 0), \varphi)$-inner amenable.

Similarly, we can prove (ii).

Corollary 3.3 Let $A$ and $B$ be two $\mathfrak{U}$-bimodule Banach algebras and $1 \leq p \leq \infty$. Then $A \oplus_{p} B$ is module $((\phi, 0), \varphi)$-inner amenable and module $((0, \psi), \varphi)$-inner amenable for every $\varphi \in \Delta(\mathfrak{U}), \phi \in \Omega_{A}$ and $\psi \in \Omega_{B}$ if and only if both $A$ and $B$ are module character inner amenable.

We note that for two Banach algebras $A$ and $B$, a direct verification shows that

$$
\Delta\left(A \oplus_{p} B\right)=(\Delta(A) \times\{0\}) \cup(\{0\} \times \Delta(B)), 1 \leq p \leq \infty .
$$

Now, if we take $\mathfrak{U}=\mathbb{C}$ and $\varphi$ is the identity map in the above corollary, then we obtain that $A \oplus_{p} B$ is character inner amenable if and only if both $A$ and $B$ are character inner amenable. Therefore, the above corollary generalizes Proposition 4.2 of [6].

Let $A$ be a Banach algebra and $X$ be a Banach $A$-bimodule. The $l^{1}$-direct sum of $A$ and $X$, denoted by $A \oplus_{1} X$, with the product defined by 


$$
(a, x)\left(a^{\prime}, x^{\prime}\right)=\left(a a^{\prime}, a \cdot x^{\prime}+x \cdot a^{\prime}\right)\left(a, a^{\prime} \in A, x, x^{\prime} \in X\right),
$$

is a Banach algebra that is called the module extension Banach algebra of $A$ and $X$.

If $A$ is $\mathfrak{Q}$-bimodule and $X$ is a Banach $A-\mathfrak{Y}$-module, then $A \oplus_{1} X$ is Banach $\mathfrak{Q}$-bimodules under the module actions:

$$
\begin{aligned}
\alpha .(a, x)= & (\alpha . a, \alpha \cdot x),(a, x) \cdot \alpha=(a . \alpha, x . \alpha) \\
& (a \in A, x \in X, \alpha \in \mathfrak{U}) .
\end{aligned}
$$

Let $A$ and $B$ be Banach algebras and let $X$ be a Banach $A, B$-module; that is, a left $A$-module and a right $B$-module satisfying $\|a x b\| \leq\|a\|\|x\|\|b\|,(a \in A, b \in B, x \in X)$. The corresponding triangular Banach algebra

$$
\tau=\left\{\left(\begin{array}{ll}
a & x \\
0 & b
\end{array}\right): a \in A, x \in X, b \in B\right\},
$$

is equipped with the usual $2 \times 2$-matrix operations and the norm

$$
\left\|\left(\begin{array}{ll}
a & x \\
0 & b
\end{array}\right)\right\|=\|a\|+\|x\|+\|b\| .
$$

This Banach algebra were introduced by Forrest and Marcoux in [7]. Note that $\tau$ can be identified with the module extension $\left(A \oplus_{1} B\right) \oplus_{1} X$, in which $X$ is considered as a $A \oplus_{1} B$-module under the operations:

$$
(a, b) \cdot x=a x, x \cdot(a, b)=x b(a \in A, b \in B, x \in X) .
$$

Furthermore, if $A$ and $B$ are two Banach $\mathfrak{O}$-bimodules and $X$ is a Banach $A \oplus_{1} B$ - $\mathfrak{A}$-module, then $\tau$ is Banach $\mathfrak{A}$-bimodules under the module actions defined as (6).

Let $\phi \in \Omega_{A}$ and define $\tilde{\phi}: A \oplus_{1} X \longrightarrow \mathfrak{A}$ by $\tilde{\phi}(a, x)=\phi(a)(a \in A, x \in X)$. Then, $\tilde{\phi} \in \Omega_{A \oplus_{1} X}$.

Using Theorem 2.2, we can routinely prove the following proposition and so we omit its proof.

Proposition 3.4 Let $A$ be $\mathfrak{A}$-bimodule and $X$ be a Banach $A$-II-module and let $\varphi \in \Delta(\mathfrak{H})$ and $\phi \in \Omega_{A}$. Then $A \oplus_{1} X$ is module $(\tilde{\phi}, \varphi)$-inner amenable if and only if there exists a bounded net $\left(a_{i}, x_{i}\right)_{i}$ in $A \oplus_{1} X$ satisfying

(i) $\varphi \circ \phi\left(a_{i}\right) \longrightarrow 1$ and $\left\|a a_{i}-a_{i} a\right\| \longrightarrow 0, \| \alpha . a_{i}-$ $a_{i} . \alpha \| \longrightarrow 0$ and $\left\|\alpha \cdot x_{i}-x_{i} \cdot \alpha\right\| \longrightarrow 0$ for all $a \in A$ and $\alpha \in \mathfrak{U}$

(ii) $\left\|x . a_{i}-a_{i} \cdot x\right\| \longrightarrow 0$ for all $x \in X$, and

(iii) $\left\|a \cdot x_{i}-x_{i} \cdot a\right\| \longrightarrow 0$ for all $a \in A$.

Corollary 3.5 Let $A$ be $\mathfrak{A}$-bimodule and $X$ be a Banach $A$ - $\mathfrak{A}$-module and let $\varphi \in \Delta(\mathfrak{I})$ and $\phi \in \Omega_{A}$. If $A \oplus_{1} X$ is module $(\tilde{\phi}, \varphi)$-inner amenable, then $A$ is module $(\phi, \varphi)$ inner amenable.

Corollary 3.6 Let Aand $B$ be $\mathfrak{A}$-bimodules and $X$ be $a$ Banach $A \oplus_{1} B$-II-module. If $\tau$ is module character inner amenable, then so are $A$ and $B$.
Proof Suppose that $\tau$ is module character inner amenable. By Corollary 3.5, $A \oplus_{1} B$ is module character inner amenable. Therefore, corollary 3.3 implies that $A$ and $B$ are module character inner amenable.

Acknowledgements We are thankful to the referees for their valuable suggestions and comments.

Open Access This article is distributed under the terms of the Creative Commons Attribution 4.0 International License (http://crea tivecommons.org/licenses/by/4.0/), which permits unrestricted use, distribution, and reproduction in any medium, provided you give appropriate credit to the original author(s) and the source, provide a link to the Creative Commons license, and indicate if changes were made.

\section{References}

1. Amini, M., Bodaghi, A., Babaee, R.: Module derivations into iterated duals of Banach algebras, Proc. Rom. Aca., Series A, 12, 277-284 (2011)

2. Amini, M., Bodaghi, A., Ebrahimi Bagha, D.: Module amenability of the second dual and module topological center of semigroup algebras. Semigroup Forum 80, 302-312 (2010)

3. Bami, M. L., Valaei, M., Amini, A.: Module amenability and weak module amenability of Banach algebras, U.P.B. Sci. Bull., Series A, 76, 35-44 (2014)

4. Bodaghi, A., Amini, M.: Module character amenability of Banach algebras. Arch. Math. 99, 353-365 (2012)

5. Bodaghi, A., Ebrahimi, H., Lashkarizadeh Bami, M., Nemati, M.: Module mean for Banach algebras, U.P.B. Sci. Bull., Series A, 78, 21-30 (2016)

6. Ebrahimi Vishki, H.R., Khoddami, A.R.: Character inner amenability of certain Banach algebras. Colloq. Math. 122, 225-232 (2011)

7. Forrest, B.E., Marcoux, L.W.: Derivations on triangular Banach algebras. Indiana Univ. Math. J. 45, 441-462 (1996)

8. Jabbari, A., Mehdi Abad, T., Zaman Abadi, M.: On $\varphi$-inner amenable Banach algebras. Colloq. Math. 122, 1-10 (2011)

9. Kaniuth, E., Lau, A., Pym, J.: On $\varphi$-amenability of Banach algebras. Math. Proc. Camp. Phil. Soc. 144, 85-96 (2008)

10. Lau, A.T.: Analysis on a class of Banach algebras with applications to harmonic analysis on locally compact groups and semigroups. Fund. Math. 118, 161-175 (1983)

11. Mun, W.D.: A class of irreducible matrix representation of an arbitrary inverse semigroup. Proc. Glasgow Math. Assoc 5, 41-48 (1961)

12. Nasr-Isfahani, R.: Inner amenability of Lau algebras. Arch. Math. (Brno) 37, 45-55 (2001)

13. Pourmahmood Aghababa, H.: (Super) module amenability, module topological centre and semigroup algebras. Semigroup Forum 81, 344-356 (2010)

14. Pourmahmood Aghababa, H.: H.: A note on two equivalence relations on inverse semigroups. Semigroup Forum 48, 200-202 (2012)

15. Rezavand, R., Amini, M., Sattari, M.H., Ebrahim Bagha, D.: Module Arens regularity for semigroup algebras. Semigroup Forum 77, 300-305 (2008)

16. Rieffel, M.A.: Induced Banach representations of Banach algebras and locally compact groups. J. Funct. Anal. 1, 443-491 (1967)

17. Schaefer, H.H.: Topological Vector Space, Springer-Verlag (1979) 